\title{
Ingenierías USBMed
}

\section{Las capacidades tecnológicas de los adultos en una comuna veracruzana}

\section{The technological capabilities of adults in a Veracruz commune}

\author{
Rosario Carvajal Hernández \\ Instituto Tecnológico Superior de San Andrés Tuxtla \\ rosariocarvajal@itssat.edu.mx \\ Erick de Jesús Téllez Vera \\ Instituto Tecnológico Superior de San Andrés Tuxtla \\ erick@itssat.edu.mx
}

\author{
Montserrat Masdefiol Suárez \\ Instituto Tecnológico Superior de San Andrés Tuxtla \\ montserratmasdefiol@itssat.edu.mx \\ María de los Ángeles Pelayo Vaquero \\ Instituto Tecnológico Superior de San Andrés Tuxtla \\ marypelayo@itssat.edu.mx
}

(Tipo de Artículo: Revisión. Recibido el 16/02/2018. Aprobado el 11/07/2018)

\begin{abstract}
Resumen. Las Tecnologías de la Comunicación y la Información (TIC) se han incorporado al entorno social, convirtiéndose en una necesidad para la vida cotidiana. El adulto como componente activo de la sociedad se ha visto involucrado en estos nuevos procesos de aprendizaje para enfrentarse al mundo contemporáneo a través del uso de estas herramientas. Estudios realizados indican que abordar estos temas son vitales para reestructurar políticas inclusivas y de desarrollo. El proyecto Alfabetización digital en un Centro de Desarrollo tuvo como objetivo determinar el impacto de alfabetización digital en adultos socialmente vulnerables mediante una Investigación tipo Investigación Acción Participativa para fungir como detonante de la inclusión social e impulsar el desarrollo de las competencias tecnológicas, una nueva cultura de la productividad, de auto-empleo y con esto contribuir a la disminución de la brecha digital.
\end{abstract}

Palabras clave. Alfabetización digital; capacidades; cultura; inclusión; tecnologías de la información y comunicación.

Abstract. The Information and Communication Technologies (ICT) have been incorporated into the social environment, becoming a necessity for daily life. The adult as an active component of society has been involved in these new learning processes to face the contemporary world through the use of these tools. Studies indicate that addressing these issues is vital to restructuring inclusive and development policies. The Digital Literacy Project in a Development Center aimed to determine the impact of digital literacy on socially vulnerable adults through a Participatory Action Research. Studies have shown that addressing these issues is vital to restructure inclusive and development policies. The Digital Literacy Project in a Development Center aimed to determine the impact of digital literacy on socially vulnerable adults through a Participatory Action Research to act as a trigger for social inclusion and promote the development of technological skills, a new culture of productivity, self-employment and thereby contribute to the reduction of the digital divide.

Keywords. Digital literacy; capacities; culture; inclusion; ICT.

DOI 10.21500/20275846.3388

\section{Introducción}

La importancia del aprendizaje y empleo de las Tecnologías de la Información y Comunicación (TIC) radica en la posibilidad de que los individuos puedan adquirir conocimientos mediante nuevas herramientas, permitiéndoles involucrarse en diversas áreas, con ello logrando mejorar su calidad de vida.

Un término que implica el uso de las TIC es el de Tecnología Educativa (TE), misma que es caracterizada por ser incluyente en la forma de insertar variadas ideologías científicas, viva por la evolución a lo largo de la historia causada por los productos de las ciencias de las que se apoya, con diversos significados de acuerdo al entorno en el que se emplea, discrepante debido a las cambiantes líneas de influencia y elocuente por la trascendencia en publicaciones [1]. Aunado a esto, el aprendizaje es un proceso que implica observación, experimentación y repetición de acciones, permitiendo efectuar de mejor forma las actividades y distinguir nuevas oportunidades a lo largo del mismo, obteniendo de este modo el conocimiento. Así, el conocimiento es resultado del aprendizaje, y cuando se adquiere éste por el uso de las TIC permite crear capacidades tecnológicas. Las capacidades tecnológicas fungen como elemento fundamental de la competitividad.

Una de las modalidades de la educación de personas adultas que emplean equipos de cómputo, que usan multimedios e Internet es con la finalidad de comunicarse con otras personas y a su vez estar 
informados, plantea como objetivo el empleo de las TIC para lograr la integración con la sociedad actual, la interacción, lazos intergeneracionales, aportaciones, premura y abogacía para continuar el aprendizaje, adiestrándose, y expresándose [2].

Actualmente las TIC forman parte primordial del entorno, llegando a convertirse en factor crucial para la vida misma, y a pesar de que éstas son útiles se debe trabajar para poder disminuir la brecha digital en algunos sectores o grupos de la población.

La brecha digital puede ser considerada como primaria cuando se relaciona con el acceso y las desigualdades económicas, mientras que se aprecia como secundaria aquella que hace referencia a la alfabetización digital [3].

Lo ya dicho, hace alusión a palabras de la reseña de la búsqueda incierta donde se plantea la exploración realizada por aquellos países en transformación por poseer métodos que permitan producir doctrinas científicas y tecnológicas que favorezcan el ascenso de la nación. La denominada "brecha" con respecto a los países destacados se ha acrecentado vertiginosamente, añadiendo con ello diversas situaciones adversas [4].

En México, el Instituto Nacional de Estadística y Geografía (INEGI), mediante la Encuesta Nacional sobre Disponibilidad y Uso de Tecnologías de la Información en los Hogares [5], menciona que el porcentaje de la población que hace uso de Internet en la provincia de Veracruz en edad de 55 o más es del $6.5 \%$, lo que indica que este sector al estar excluidos al uso de TIC, para socializar, comunicarse, quedan aislados provocando que sean consideradas como personas inactivas. Sin embargo, la Organización Mundial de la Salud, define envejecimiento activo como "el proceso de optimización de las oportunidades de salud, participación y seguridad, con el fin de mejorar la calidad de vida a medida que las personas envejecen" [6].

El adulto que no posee conocimientos informáticos no es un componente activo de la sociedad y éste se encuentra involucrado en nuevos procesos de aprendizaje y se confrontará al mundo interconectado, con el uso de las tecnologías en mención, puesto que éste sector de la población ha sufrido repentinos cambios en cuanto a su relación con las mismas, por esta razón es inminente que deben ser generados mecanismos que puedan contribuir a la formación de conocimientos que les concedan interactuar con dichas tecnologías.

La alfabetización se encuentra acoplada a dos elementos: entorno de la familia y las instituciones educativas, ella sirve para continuar el mencionado proceso y establecer la relación entre los elementos ya indicados a través del respeto y la enseñanza. Es cierto que la falta de continuidad en las actividades escolares que favorecen el aprendizaje perjudica la adquisición del conocimiento. Sin embargo, buscando mejorar la calidad en la enseñanza se deben incluir formación
Vol. 9 (2), Pág. 44-49, julio-diciembre 2018

personalizada, tiempo, equipo y facilidad para profundizar y descubrir [7].

La alfabetización digital es el cúmulo de capacidades socio cognitivas a través de las cuales es posible escoger, procesar, examinar y notificar el proceso de evolución de información al conocimiento a través de equipos inherentes a las TIC [8].

En ese contexto, la capacidad tecnológica es la aptitud de poder contar con una perspectiva globalizada de los elementos tecnológicos en el mercado, obtener su valor, elegir la tecnología acorde a las necesidades, emplearla, ajustarla, corregirla y en la última etapa desarrollarla. Esta capacidad es posible que la posean desde los productores directos (agricultores, trabajadores), hasta los encargados de tomar decisiones en las empresas, en agencias estatales, etc. [9].

Capacidad tecnológica no es exclusivamente poseer la tecnología requerida para preparar un producto determinado. Este concepto alude a todo ese conjunto de acciones que generan la posibilidad de que la institución cuente con la ventaja de seleccionar y emplear tecnología, para crear utilidades competitivas [10].

Así mismo, es posible asegurar que una capacidad tecnológica es estar preparado para comprender realmente la cultura tecnológica [11]. Se mencionan algunas habilidades tecnológicas, tales como: ejercicios prácticos empleando herramientas informáticas, redacción de textos (procesador de palabras), mecanización de cálculos matemático (hojas de cálculo), multimedia (programa de presentaciones), lista de clientes (bases de datos), servicios, software y aplicaciones vinculadas con Internet, etc.

Aunado a lo anteriormente mencionado, las instituciones de educación superior juegan un papel importante en el desarrollo de la zona de influencia donde se encuentran ubicados, tal es que los esquemas actuales del binomio instituciones de educación superior y sociedad están fundamentados en la premura de colaborar de manera conjunta con la finalidad de satisfacer en cierta medida las necesidades que presenta está ultima, éste binomio debe ser apoyado por diversos entes, tales como gobierno tanto local como federal, bibliotecas, empresas, lideres, etc. Las instituciones de educación superior deben fungir como facilitadores del conocimiento y originalidad [12].

En resumen, el presente trabajo específica el diagnóstico de las capacidades tecnológicas que expone la población adulta inexperta en el área de informática de un sector de la población de una zona urbana al sureste de la provincia de Veracruz, México, mediante la aplicación del instrumento de medición como lo es el cuestionario.

\section{Desarrollo de la investigación}

La determinación del nivel de uso de las TIC en la población adulta de un sector de la población de una comuna de la provincia de Veracruz, México, es una 
investigación tipo Investigación Acción Participativa (IAP), así como Exploratoria - Cuantitativa, con el objetivo de establecer el grado de atraso digital, para posteriormente implementar diversas estrategias con la finalidad de reducir éste, ya que debido a ello, se modificó el enfoque de enseñanza, puesto que en otra época estaba encauzado a la formación, en contraste, en la actualidad se encamina hacia el aprendizaje, siendo la causa del acontecer del aprendizaje cimentado en las competencias, ya que éste último, se enfila a los conocimientos, capacidades y valores [13].

El proceso de desarrollo fue durante el periodo de tiempo entre septiembre de 2016 y abril de 2017, llevando a cabo una serie de fases, las cuales se engloban en tres etapas que componen a la IAP [14], las cuales se mencionan en la Tabla 1.

Tabla 1 Etapas y fases de la IAP

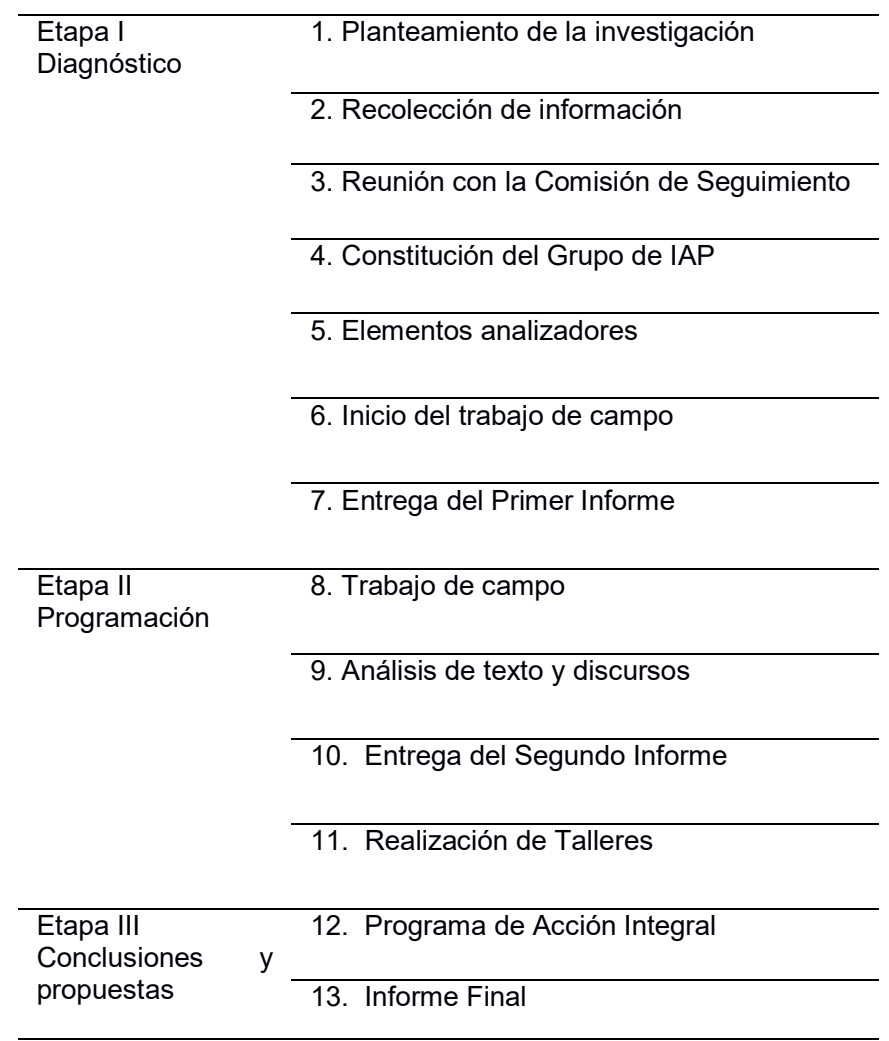

De manera general, el proyecto de investigación se basó en un estudio para promover y acondicionar un Centro de Capacitación Digital en conjunto con la Dirección de Desarrollo Social de una comuna veracruzana, con el objetivo de fomentar la alfabetización digital e impulsar las competencias digitales, promover la productividad laboral y de autoempleo en las personas adultas con grado de marginación alto. Identificada la demanda de estudio, se realizó el diagnóstico y acercamiento a la problemática a partir de entrevistas y aplicación de instrumentos de recolección de datos para obtener datos precisos sobre la cantidad de analfabetas digitales existentes, mediante el apoyo del equipo de investigación integrado por personas tanto de la comuna como de la institución de educación superior.
Ingeniería USBMed Vol. 9 (2), Pág. 44-49, julio-diciembre 2018 3. Planteamiento del problema

La comuna en estudio está situada aproximadamente a $150 \mathrm{~km}$ al sureste de la provincia de Veracruz, es la mayor y más poblada comuna en la Sierra de Los Tuxtlas, de acuerdo a datos publicados por el Instituto Nacional de Estadística y Geografía (INEGI) del registro de población del año 2010 [15], en la comuna en comento, hay una población de 157,364 , de la cual la mitad de la población tiene 25 años o menos. Está integrada de 261 localidades y 70 colonias, tiene como actividad de ingreso base el sector primario, el cual aunado a las expectativas de búsqueda de nuevas oportunidades se genera migración, concentración e incremento de la población urbana demandantes de empleo, bienes y servicios.

En el sector educacional de la comuna en estudio, la población mayor de 15 años de edad, considerado como adultos para el país, con respecto al grado de escolaridad muestra que el $6.8 \%$ no posee nivel escolar alguno y el $62 \%$ únicamente cuenta con escolaridad principal; quiere decir que aproximadamente el $70 \%$ de la sociedad presenta un rezago educativo.

EI INEGI indica que, con respecto a las TIC, el $47 \%$ de los domicilios notifican contar con servicio de tecnología celular, $11 \%$ posee una computadora y $6 \%$ emplean el servicio de Internet.

El Plan Municipal de Desarrollo (PMD) es el mecanismo principal en el que se indican las políticas y actividades públicas de la administración municipal que están encaminadas a lograr el mayor bienestar de todos los ciudadanos.

Para el actual Gobierno municipal de la comuna en estudio, es fundamental tomar decisiones encaminadas a cubrir las necesidades de la ciudadanía e impulsar la educación tecnológica, basándose en una cultura de justicia, equidad, étnica, de género e inclusión.

Con los resultados del Plan Maestro del Programa Hábitat (PMPH) y las gestiones realizadas por el $\mathrm{H}$. Ayuntamiento de la mencionada comuna y a través de la Secretaría de Desarrollo Agrario, Territorial y Urbano (SEDATU), se construyó un Centro de Desarrollo para los habitantes de las colonias con un alto grado de pobreza y marginación, con la finalidad de implementar cursos y talleres en beneficio del desarrollo de las familias y atención al maltrato de la niñez, abatir la drogadicción, alcoholismo y actividades deportivas.

La comuna veracruzana presenta un polígono de pobreza conformado por las diversas colonias circunscritas al Centro de Desarrollo, lugar constituido para ofertar cursos y talleres a los ciudadanos de la zona de estudio con la finalidad de instruirlos en diversas temáticas en las que adolecen de conocimientos, especialmente en capacidades tecnológicas. La primordial forma de obtención de recursos económicos parten de actividades derivadas del cultivo y diversos oficios como carpintero, mecánico, albañil, plomero, pintor de brocha gorda, vendedor, vigilante, chofer, 
peluquero y frutero, así como personas jubiladas; debido a lo anterior, tienen un nivel de vida austero con respecto a los ingresos obtenidos, en este país, la economía familiar cuenta con un aproximado a los 12,806 USD anualmente, cantidad inferior que el estipulado por la Organización para la Cooperación y el Desarrollo Económicos de 29,016 USD en ese periodo de tiempo [16].

¿Cómo determinar el nivel de uso de las TIC que poseen los adultos en una comuna veracruzana?

De acuerdo a la situación que se evidencia en el lugar en mención, se determina que las hipótesis son las siguientes:

Ho: A través del dictamen de las aptitudes tecnológicas de los adultos de una comuna veracruzana, no se podrá establecer el grado de uso de las TIC.

$\mathrm{Hi}$ : A través del dictamen de las aptitudes tecnológicas de los adultos de una comuna veracruzana, se podrá establecer el grado de uso de las TIC.

Para efectos de esta investigación la población con la que se trabajó para diagnosticar las capacidades tecnológicas y determinar capacidades en el uso de las TIC fue una colonia de la comuna ubicada al sureste de la provincia veracruzana, la cual cuenta con una población de 1550 habitantes, se determinó la muestra estadística, obteniendo un valor de 65 con un margen de error de $10 \%$ y nivel de confianza de $90 \%$.

La recolección de datos fue efectuada mediante un cuestionario impreso de dieciocho ítems, donde los indicativos empleados para establecer el grado de usos de las TIC se encuentran: aptitudes tecnológicas, empleo de dispositivos tecnológicos, empleo de aplicaciones de oficina y uso de software de Internet.

El resultado generado de la aplicación del instrumento de medición se efectuó con el software de IBM SPSS Statistics Base empleado en la toma decisiones, mejorando la eficiencia y minimizando el riesgo de errores, con los siguientes resultados: el $60 \%$ de las personas encuestadas son de sexo masculino y el $40 \%$ femenino, la población de la colonia de la comuna cuenta predominantemente con habitantes de edad promedio de 31 a 40 años, siendo la ocupación principal las amas de casa y trabajadores, la mayoría de estas personas poseen un nivel de estudio de educación básica, lo anterior se representa en la Figura 1.
Vol. 9 (2), Pág. 44-49, julio-diciembre 2018

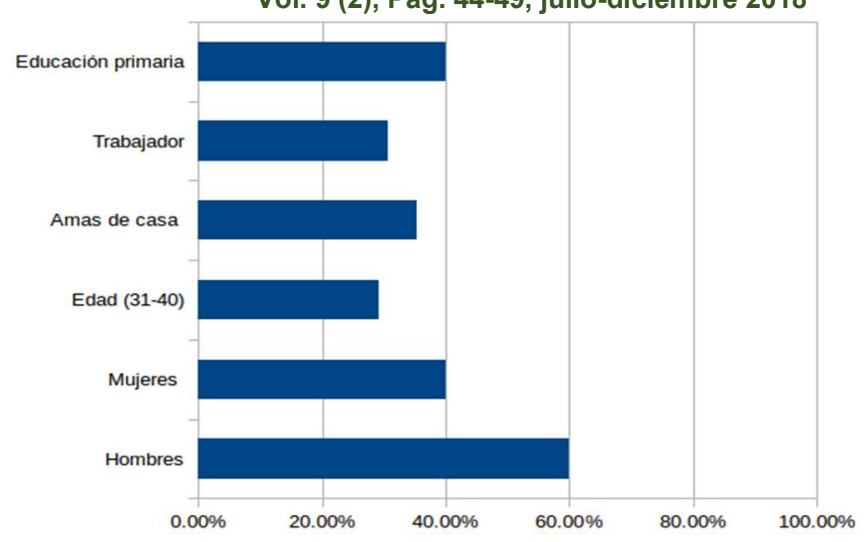

Figura 1. Información básica de la comuna.

\subsection{Resultados}

Derivado del instrumento de recolección de datos, los indicadores empleados para conocer el grado de empleo de las TIC se tiene en primera instancia a las capacidades tecnológicas, los datos que a continuación se mencionan se derivan de la muestra estadística utilizada, donde el $40 \%$ utiliza con frecuencia la computadora con la finalidad de elaborar tareas y navegar en Internet son jóvenes de 15 a 25 años, el $46.15 \%$ son personas mayores de 25 años que utilizan en su mayoría la Tablet y Smartphone, además indican que utilizan el Internet todos los días principalmente para accesar a las redes sociales sin contar con una preparación académica del uso del Internet. Lo anteriormente mencionado se representa en la Figura 2.

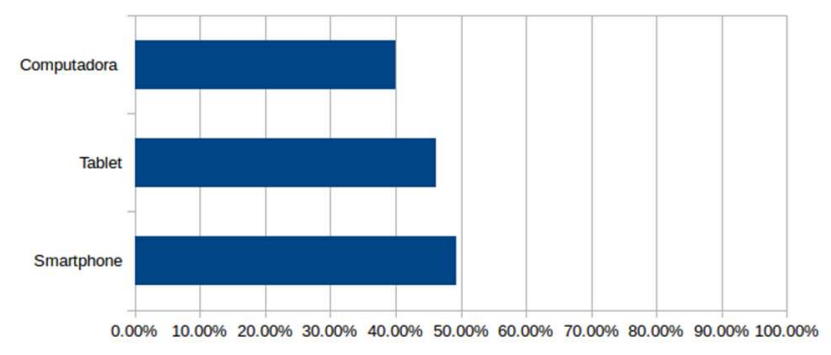

Figura 2. Indicador: capacidades tecnológicas.

Con respecto al uso de dispositivos tecnológicos, el $44.62 \%$ de la muestra aprendieron a usar el Internet por ellos mismos, demostrando interés en aprender más acerca del uso de esta tecnología. En otro aspecto evaluado, un $63.08 \%$ de la muestra no pueden realizar trabajos de computadora por sí mismos por analfabetismo digital y el $36.92 \%$ del $86.15 \%$ considerada como población adulta de la muestra en estudio tienen enseñanzas básicas del manejo de la computadora, lo que les permite utilizarla con mayor frecuencia. Es así como en la Figura 3 se encuentra de manera gráfica esta información. 


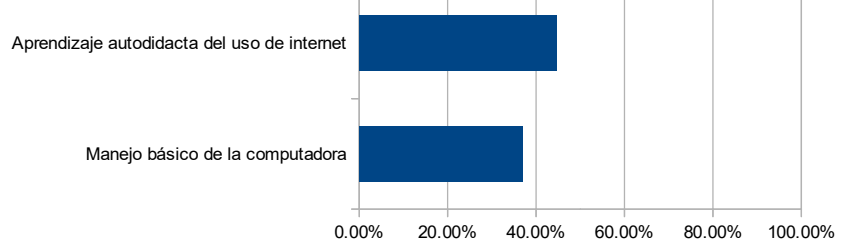

Figura 3. Indicador: uso de dispositivos tecnológicos.

En contraparte, en el uso de software de oficina, la mayoría no utilizan el procesador de texto ni las hojas de cálculo debido a que adolecen de la capacitación, la minoría emplea éstos de manera autodidacta para realizar operaciones en sus trabajos u hogares. Así mismo, el $22.15 \%$ del $17 \%$ de la persona que poseen una computadora portátil o de escritorio de la muestra realizan presentaciones en sus equipos de cómputo. La Figura 4 ilustra dichos porcentajes.

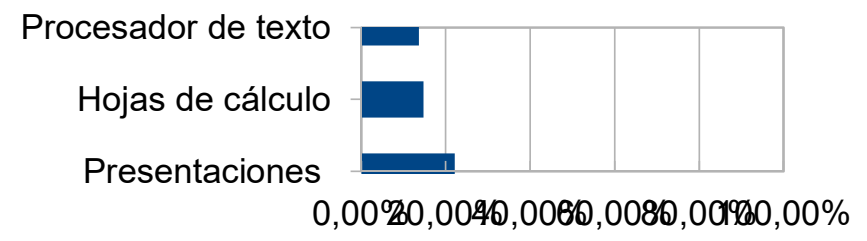

Figura 4. Indicador: uso de software ofimático.

Finalmente, con respecto al uso de Internet, el $19.16 \%$ de la población que posee el servicio de Internet en casa que es el $38.92 \%$ del $86.15 \%$ de las personas adultas de la muestra, hace uso de la banca electrónica, lo cual les facilita realizar operaciones de transferencia y consulta, el $15.57 \%$ respectivamente realizan trámites administrativos, el resto no cuenta con el servicio de Internet. Por otra parte, la mayoría de las personas no hacen uso de navegadores por miedo a que puedan ser infectados sus dispositivos con algún virus, igualmente las personas prefieren utilizar el correo como una alternativa para enviar tareas o trabajos y hacer uso de documentos, el resto no hacen uso de éste por falta de conocimiento. Sin embargo, la mayor parte de la población hace uso de las redes sociales, en especial Facebook para estar informados, así como conectados con familiares o amigos, lo anterior se visualiza en la Figura 5.

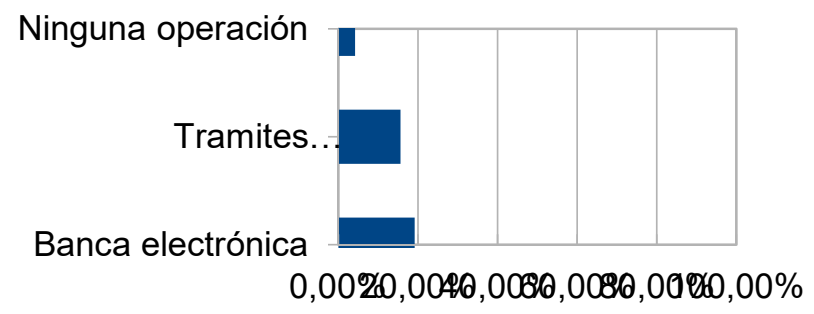

Figura 5. Indicador: Uso de Internet.

\section{Resumen de resultados}

En la culminación de este proyecto es donde se logra convenir el grado de las aptitudes en el uso de las TIC de una colonia perteneciente a una comuna de la provincia de Veracruz, México, la investigación incluyó el empleo de un instrumento de recolección de datos como lo es el cuestionario, mismo que se aplicó a una muestra estadística de 65 ciudadanos adultos. Con respecto al uso de los dispositivos tecnológicos como variable dependiente evidencian que el $4 \%$ saben utilizar computadora/laptop, el $4 \%$ saben utilizar la Tablet, además un $4.92 \%$ utiliza Smartphone y el $4.30 \%$ navega por Internet teniendo la cifra de $41.83 \%$, lo que se considera escaso.

Con respecto a la variable independiente, compete al grado de uso de las TIC, es notable que el indicativo del uso de dispositivos tecnológicos muestra que el $8.2 \%$ de un total de $22.22 \%$ de la población encuestada saben utilizar diversos dispositivos tecnológicos. Por otra parte, en el indicador de uso de software de ofimática se encontró que el $5.07 \%$ de $33.33 \%$ esperado tiene este tipo de conocimiento, lo cual es insuficiente ya que no rebasa el $50 \%$ de la cifra considerada. Con referencia el indicador de uso de Internet el $7.39 \%$ de los $44.44 \%$ tienen la capacidad de manipular software de Internet, cifra que es insuficiente ya que no rebasa el $50 \%$. Esto quiere decir que las personas se enfocan más en el uso de dispositivos tecnológicos que pueden favorecer y agilizar su vida cotidiana.

Efectuando una compilación de los datos los indicativos analizados en la variable grado de uso de las $\mathrm{TIC}$, se tiene un valor de $20.66 \%$, al contrastarlo con una tabla de valores es una cota escueta.

\section{Conclusiones}

Como desenlace de la investigación, se determina que la población de la comuna estudiada tiene un alto índice de pobreza, debido a que la mayoría de los pobladores sólo tienen la instrucción básica, además de no contar con los recursos que les permitan desarrollar sus competencias tecnológicas. Por lo tanto, requieren de capacitación para incrementar el índice de alfabetización digital y de esta manera poder contribuir a la disminución de la brecha digital existente.

El Centro de Desarrollo en conjunto con una Institución de educación superior de la región y el Honorable Ayuntamiento de la comuna, deberán diseñar e implementar un programa de capacitación que incorpore cursos en los que se fomenten las capacidades y crecimiento de competencias en el empleo de las TIC, para garantizar a la población adulta la adquisición de conocimientos y destrezas en el uso de las nuevas tecnologías. Ambas instituciones deberán motivar a las personas de las colonias circunscritas a mencionado Centro para que por medio de los talleres ofertados de manera gratuita obtengan mayores oportunidades de desenvolverse en ámbito social y laboral.

A manera de resumen acorde a los datos recolectados se plantea una serie de cursos que la población estudiada requiere, tales como: uso básico de una computadora, uso del sistema operativo, software 
de oficina y manejo básico de Internet, cabe mencionar que la capacitación debe ser formal y acorde a un plan pedagógico que ayude a esta población marginada.

\section{Trabajos futuros}

Se aconseja trabajar en el diagnóstico de las capacidades en las otras colonias circunscritas al Centro de Desarrollo para que se pueda incrementar el índice de alfabetización digital en la población socialmente vulnerable. Podríamos sugerir que hay un abundante campo todavía por explorarse en lo que se refiere a alfabetización digital, ya que una vez efectuado el dictamen en mencionado polígono, proceder a diseñar conforme a las necesidades detectadas los cursos de capacitación que serán impartidos en dicho Centro para con ello poder contrastar el impacto de las acciones implementadas.

Asimismo, se recomienda al ayuntamiento de la comuna mejorar el acceso a Internet en las zonas de estudio, ya que solo se cuenta con una empresa proveedora siendo ésta por telefonía fija, así como gestionar apoyos con lo que se pueda dotar de equipos referentes a las TIC.

\section{Agradecimientos}

Agradecen a V. Guerrero Hernández (México) las ayudas en la fijación de los criterios lingüísticos.

\section{Referencias}

[1] J. Cabero, "Tecnología Educativa. Diseño y utilización de medios en la enseñanza". Barcelona. Paidós, 2001.

[2] F. Pavón, "Nuevas tecnologías aplicadas al aprendizaje adulto". En Cebrian, M. y Ríos, J.M. (Coords.) Nuevas tecnologías aplicadas a las didácticas especiales. Madrid, Pirámide, pp. 221-238. 2000.

[3] F. Colombo, P. Aroldi, S. Carlo, "Nuevos mayores, viejas brechas: TIC, desigualdad y bienestar en la tercera edad en Italia". En Comunicar: Revista Científica de Comunicación y Educación, Vol. 23, núm (45). . págs. 47-55, 2015.

[4] P. Kreimer, Reseña de "La búsqueda incierta. Ciencia, Tecnología y Desarrollo", Redes, vol. 4, núm. 10, octubre, 1997, pp. 259-263.
[5] ENDUTIH, INEGI. (2017, marzo, 18). Encuesta Nacional sobre Disponibilidad y Uso de Tecnologías de la Información en los Hogares 2017 [Online]. Available: http://www.beta.inegi.org.mx/proyectos/enchogares/reg ulares/dutih/2017/

[6] M. Sevilla Caro, M. del C. Salgado Coto, N. del C. Osuna Millán, Envejecimiento activo. Las TIC en la vida del adulto mayor, Revista iberoamericana para la investigación y el desarrollo educativo. Vol. 6, Núm. 11. 2015.

[7] B. Braslavsky, "Las nuevas perspectivas de la alfabetización temprana". En Lectura y Vida, 2000.

[8] P. Gilster, "Introduction to Excerpt", Digital Literacy, Londres, John Wiley and Sons, 1997.

[9] J. Meyer-Stamer. (2017, septiembre 02) "Mesopartner" [Online]. Available: http://www.meyer-stamer.de

[10] World Bank, "Innovation Capacity Tool: Firm level innovation in the Korean economy", Innosutra, 2001, p10.

[11] V. A. Vázquez. (2017, septiembre 02). Habilidades tecnológicas [Online]. Available: https://www.entrepreneur.com/article/255332,

[12] C. A. Hernández, "Roles de los centros universitarios municipales en el desarrollo local de los municipios cubanos", Revista Ingenierías USBMed, Vol. 3, No. 1. 2012.

[13] A. Lagunes Domínguez, "El camino de la innovación educativa y su encuentro con las ingenierías", Revista Ingenierías USBMed, Vol.8, Pag.4-6, ISSN: 2027-5846, año 2017.

[14] J. Martí, La investigación-acción-participativa: Estructuras y fases. In T. Rodríguez Villasante, M. Montañez, \& J. Martí (Coords.), La investigación social participativa: Construyendo ciudadanía.Vol. 1, pp. 79123. Madrid: El Viejo Topo. 2002.

[15] INEGI. (2017, marzo 18) Censo de Población y Vivienda 2010

[Online]. Available:http://www.beta.inegi.org.mx/proyectos/ccpv/ 2010/default.html

[16] OCDE. (2017, agosto 29) México, ¿Cómo es la vida? [Online]. Available: http://www.oecdbetterlifeindex.org/es/countries/mexicoes/ 